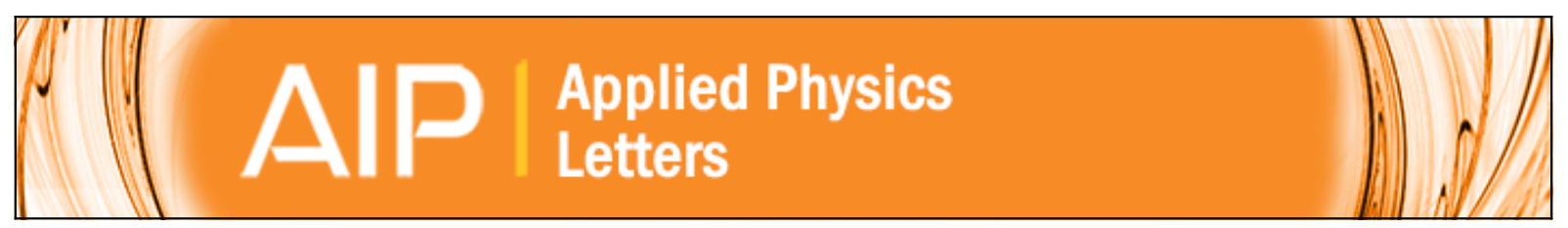

\title{
Band offsets at zincblende-wurtzite GaAs nanowire sidewall surfaces
}

P. Capiod, T. Xu, J. P. Nys, M. Berthe, G. Patriarche, L. Lymperakis, J. Neugebauer, P. Caroff, R. E. DuninBorkowski, Ph. Ebert, and B. Grandidier

Citation: Applied Physics Letters 103, 122104 (2013); doi: 10.1063/1.4821293

View online: http://dx.doi.org/10.1063/1.4821293

View Table of Contents: http://scitation.aip.org/content/aip/journal/apl/103/12?ver=pdfcov

Published by the AIP Publishing

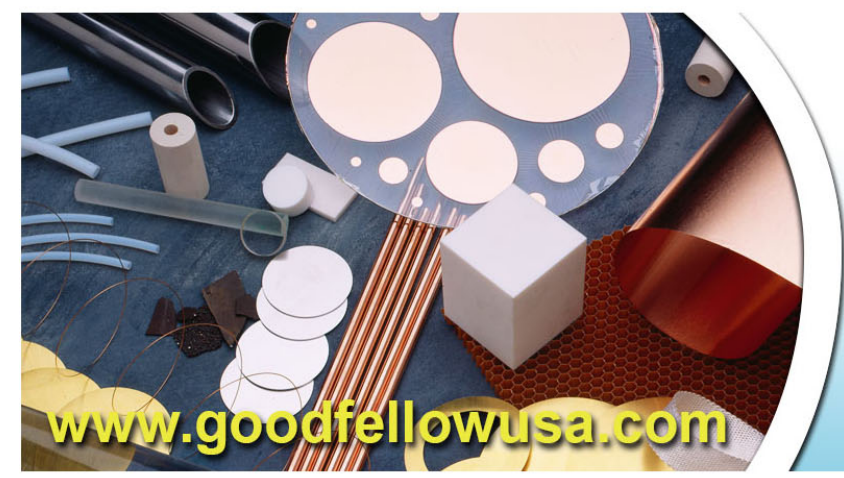

BDOdFE/|III

metals • ceramics • polymers composites • compounds • glasses

Save $5 \% \cdot$ Buy online 70,000 products $\cdot$ Fast shipping 


\title{
Band offsets at zincblende-wurtzite GaAs nanowire sidewall surfaces
}

\author{
P. Capiod, ${ }^{1}$ T. Xu, ${ }^{1,2}$ J. P. Nys, ${ }^{1}$ M. Berthe,${ }^{1}$ G. Patriarche,${ }^{3}$ L. Lymperakis, ${ }^{4}$ J. Neugebauer, ${ }^{4}$ \\ P. Caroff, ${ }^{1,5}$ R. E. Dunin-Borkowski, ${ }^{6}$ Ph. Ebert, ${ }^{6}$ and B. Grandidier ${ }^{1}$ \\ ${ }^{1}$ Institut d'Electronique et de Microélectronique et de Nanotechnologies, IEMN, CNRS, UMR 8520, \\ Département ISEN, 41 bd Vauban, 59046 Lille Cedex, France \\ ${ }^{2}$ Key Laboratory of Advanced Display and System Application, Shanghai University, 149 Yanchang Road, \\ Shanghai 200072, People's Republic of China \\ ${ }^{3}$ CNRS-Laboratoire de Photonique et de Nanostructures (LPN), Route de Nozay, 91460 Marcoussis, France \\ ${ }^{4}$ Max-Planck Institut für Eisenforschung GmbH, Max-Planck Str. 1, 40237 Düsseldorf, Germany \\ ${ }^{5}$ Department of Electronic Materials Engineering, Research School of Physics and Engineering, \\ The Australian National University, Canberra ACT 0200, Australia \\ ${ }^{6}$ Peter Grünberg Institut, Forschungszentrum Jülich GmbH, 52425 Jülich, Germany
}

(Received 31 July 2013; accepted 26 August 2013; published online 18 September 2013)

\begin{abstract}
The band structure and the Fermi level pinning at clean and well-ordered sidewall surfaces of zincblende (ZB)-wurtzite (WZ) GaAs nanowires are investigated by scanning tunneling spectroscopy and density functional theory calculations. The WZ-ZB phase transition in GaAs nanowires introduces $p-i$ junctions at the sidewall surfaces. This is caused by the presence of numerous steps, which induce a Fermi level pinning at different energies on the non-polar WZ and ZB sidewall facets. C 2013 AIP Publishing LLC. [http://dx.doi.org/10.1063/1.4821293]
\end{abstract}

In semiconductor materials, the pinning of the Fermi level at the surface together with the condition of charge neutrality can be used to modify and control the space charge at the surface. For example, by doping or introducing extrinsic surface states, the position of the Fermi level at the surface can be shifted, with dramatic effects on physical and chemical properties of the surface and the underlying material. Even though these shifts are commonly small, they strongly alter the dopant and defect incorporation ${ }^{1,2}$ and also the diffusion processes at surfaces or across interfaces. ${ }^{3-6}$

Such shifts in the Fermi level pinning cause even more pronounced modifications of the material properties at the nanoscale. Replacing donor by acceptor impurities in InP nanowires (NWs) redshifts the NW optical emission due to different band bending at the NW surface. ${ }^{7}$ Similarly, $n$ - and $p$-doped Ge NWs show a different chemical reactivity at the surface, ${ }^{8}$ with a significant change of their conductivity with respect to nominally undoped NWs. ${ }^{9}$

Recent advances in the growth of nanowires allow the fabrication of complex crystal structures, which otherwise are unstable and hence cannot be achieved in the bulk. ${ }^{10-13}$ In these semiconductor polytype materials, understanding the energetic position of surface states and Fermi level position at the surface is critical, since these parameters might govern the material transport and optical properties. ${ }^{14-16}$ However, the importance of the surface has been put aside so far, due to controversies that already exist for the band alignment in the bulk polytypes. A prototypical material is $\mathrm{GaAs}$, where polytype inclusions consisting of zinc-blende (ZB) and wurtzite (WZ) segments form during the growth of NWs and where the band discontinuities at the interface are strongly debated. ${ }^{17-20}$

In this work, we thus investigate the surface band structure and the pinning of the Fermi level position of clean and well-ordered sidewalls of GaAs NWs containing ZB and WZ phases. We demonstrate that ZB-WZ structures on the surface of GaAs NWs naturally introduce $p-i$ junctions solely due to Fermi level pinning at different energies on the non-polar WZ and ZB sidewall facets. This effect is independent of the polarization field at the interface and should apply to many different $\mathrm{ZB} / \mathrm{WZ}$ homomaterials, since it is caused by the presence of numerous steps, which are almost inevitably present on NW sidewalls.

For our experiments, we grew undoped GaAs NWs consisting of ZB and WZ segments by solid source molecular beam epitaxy. We used a natural self-overgrowth process in Ga-assisted GaAs/GaAs ${ }_{0.81} \mathrm{Sb}_{0.19} / \mathrm{GaAs} \mathrm{NW}$ heterostructures, nucleated on native oxide-covered $\mathrm{Si}(111)$ substrates, as described previously. $^{21}$ When switching from $\mathrm{GaAs}_{0.81} \mathrm{Sb}_{0.19}$ to GaAs the upper GaAs section will grow first in $\mathrm{ZB}$ structure and then switch to the WZ structure. ${ }^{21,22}$ The nanowires were grown at $630^{\circ} \mathrm{C}$ using $\mathrm{Ga}, \mathrm{As}_{4}$, and $\mathrm{Sb}_{2}$ fluxes corresponding to two-dimensional equivalent growth rates of $0.25,0.50$, and 0.66 monolayer $(\mathrm{ML}) / \mathrm{s}$, respectively. At the end of the growth, the NWs were capped with a thin As layer, deposited at room temperature for $50 \mathrm{~min}$ under high $\mathrm{As}_{4}$ flux to protect the NWs from air exposure (oxidation). ${ }^{23}$ The NWs were analyzed with transmission electron microscopy (TEM) and low-temperature scanning tunneling microscopy (STM) in ultrahigh vacuum. Prior to the STM measurements, the capping layer was desorbed at $350{ }^{\circ} \mathrm{C}$ for $4 \mathrm{~h}$, monitoring the As desorption with mass spectrometry. Then the NWs were cleaved off in situ and deposited on a freshly prepared degenerately doped $\mathrm{Si}(111)$ surface, onto which the cleaved-off NWs lie flat. ${ }^{24}$

Figure 1(a) shows a high angular dark field scanning transmission electron microscopy (HAADF-STEM) image of the $\mathrm{GaAs} / \mathrm{GaAs}_{0.81} \mathrm{Sb}_{0.19} / \mathrm{GaAs}$ (axial) heterostructured NWs. The intermediate $\mathrm{GaAs}_{0.81} \mathrm{Sb}_{0.19}$ inclusion corresponds to the bright segment. The [ $\overline{1} \overline{1} \overline{1}] \mathrm{B}$ ([000 $\overline{1}]$ for WZ segment) growth direction of the three NWs shown is toward the right side. At the top (right end), the Ga droplet used to assist growth of the nanowires is barely visible, having a slightly rounded shape, due to the incorporation of $\mathrm{Ga}$ at the end of the NW in the presence of excess arsenic during cooling 

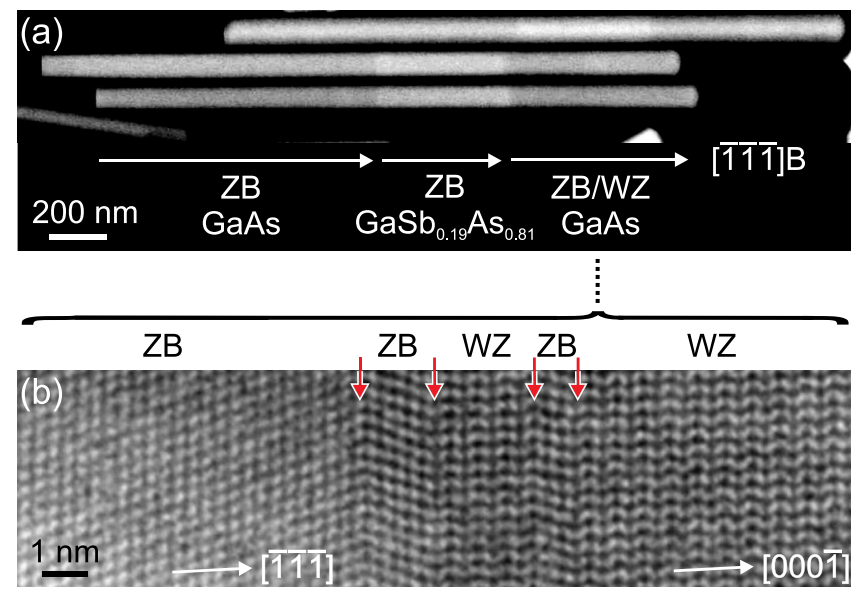

FIG. 1. (a) HAADF STEM image of GaAs/ $\mathrm{GaAs}_{0.81} \mathrm{Sb}_{0.19} / \mathrm{GaAs}$ nanowire heterostructure. The arrows define the end of the different segments and point to the growth direction. (b) Atomic resolution HAADF-STEM image of the top segment acquired in the region of the phase transition between the $\mathrm{ZB}$ and the WZ structure. The (red) arrows mark the positions of stacking faults and twin boundaries in the transition region.

down under $\mathrm{As}_{4}$ flux and the capping layer formation. ${ }^{25}$ The bottom GaAs segment (left side) and the $\mathrm{GaAs}_{0.81} \mathrm{Sb}_{0.19}$ segment grow with a perfect $\mathrm{ZB}$ structure containing no twin plane or stacking fault. ${ }^{21,25}$ In contrast, a phase transition occurs in the top GaAs segment for most NWs. Such a transition is clearly visible in the atomic resolution STEM image in Fig. 1(b), which shows a few stacking faults/twin boundaries before the growth of GaAs in the pure WZ crystal structure starts.

Aware of the existence of a transition between the ZB and the WZ structure at the top of the NWs, the morphology of the NW sidewalls was thus investigated by STM. The NWs have a hexagonal shape with six sidewalls. When one of the sidewalls is in contact with the Si substrate, the opposite sidewall is almost parallel to the scanning plane, due to the constant diameter of the NWs along the axial direction. Figure 2(a) shows a STM image acquired in the region corresponding to the upper GaAs segment. A large section of a parallel sidewall is visible. Its edges are imaged as two lines running parallel to the growth direction (see dashed lines), where the contrast changes from bright to dark as the tip scans over the adjacent and tilted sidewalls. This change is less pronounced at the bottom of the STM image due an asymmetric shape of the tip. Focusing on the top (parallel to the substrate) sidewall, we note a clear difference in the morphology of the terraces between the left and right parts of the image. To the left, the flat terraces are defined by step edges along two preferential directions, the [011] and [001] directions, consistent with ZB structure GaAs $\{110\}$ planes. ${ }^{23}$ This crystallographic orientation is confirmed with a high resolution STM image (Fig. 2(b)), which shows the anion sublattice consisting of parallel atomic rows, making an angle of $35.3^{\circ}$ with the $\langle 111\rangle \mathrm{B}$ growth axis. In the right part of Fig. 2(a), the terraces are horizontally elongated and much narrower, with the step edges propagating along the growth direction. Such a morphology is typical of the facets observed on cleaved WZ crystals or on the sidewalls of WZ NWs. ${ }^{26,27}$ Imaging the terraces at high resolution reveals the existence of zigzag rows, a signature of WZ structure
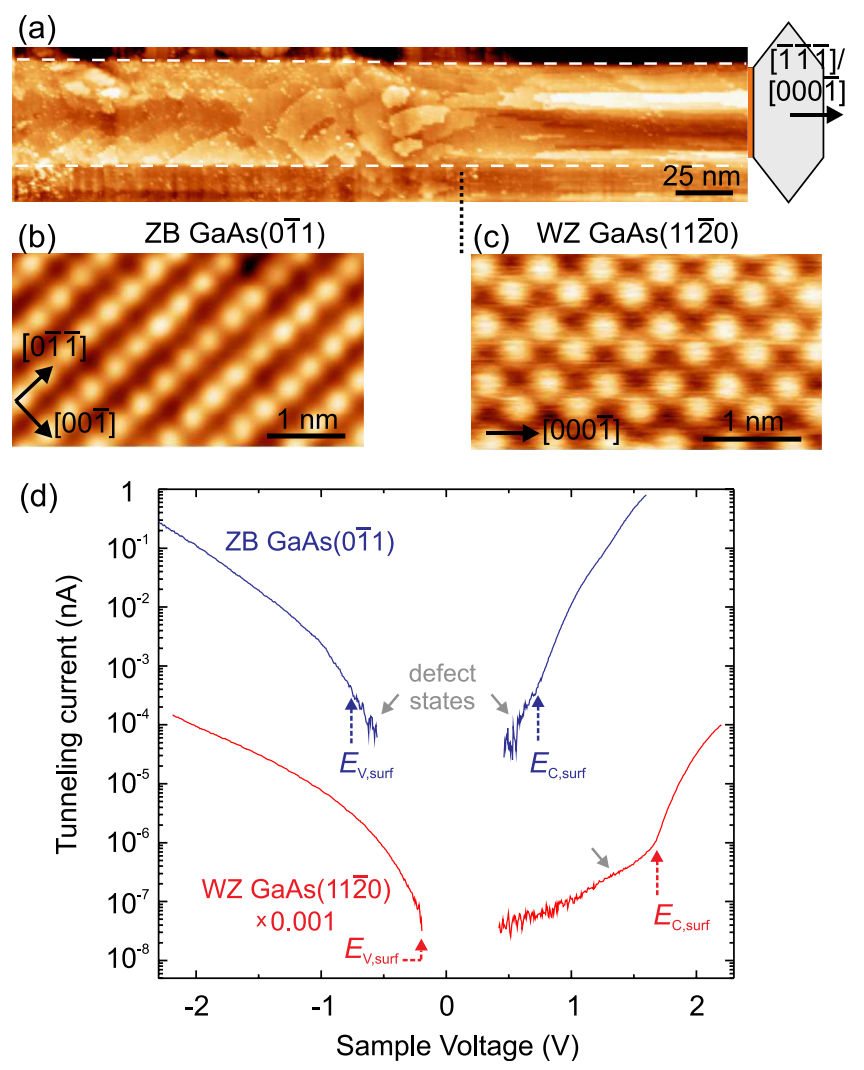

FIG. 2. (a) Large-scale STM image of a sidewall acquired on the top GaAs segment of a GaAs/ $\mathrm{GaAs}_{0.81} \mathrm{Sb}_{0.19} / \mathrm{GaAs}$ nanowire heterostructure measured at $77 \mathrm{~K}$ with a set voltage of $+2.8 \mathrm{~V}$ and $10 \mathrm{pA}$ tunnel current. The dashed lines mark the edges of the (top) sidewall parallel to the substrate. The inclined hexagon to the right illustrates the cross-sectional shape of the NW. The dotted line marks the approximate position of the ZB (left) and WZ (right) interface. (b) and (c) High resolution STM images of the GaAs ZB and $\mathrm{WZ}$ phases observed on the sidewall of the top GaAs segment measured at 20 and $100 \mathrm{pA}$ and -3.5 and $+2.0 \mathrm{~V}$, respectively. (d) Tunneling spectra acquired on the GaAs $\mathrm{ZB}$ and $\mathrm{WZ}$ phases at a temperature of $77 \mathrm{~K}$. The spectrum of the WZ phase is offset by a factor of 0.001 for clarity. The dashed arrows mark the onsets of the filled ( $\left.E_{\mathrm{V} \text {,surf }}\right)$ and empty $\left(E_{\mathrm{C} \text {,surf }}\right)$ surface states. The Fermi energy of the surfaces is at $0 \mathrm{~V}$.

$\operatorname{GaAs}(11 \overline{2} 0)$ (also called $a$-plane) sidewalls that are known to be the counterpart of the ZB $\{110\}$ facets for NWs with a growth axis along the equivalent $[\overline{1} \overline{1} \overline{1}] /[000 \overline{1}]$ directions. ${ }^{28}$

Tunneling spectroscopic measurements were performed on the sidewalls of both the $\mathrm{WZ}$ and $\mathrm{ZB}$ segments, far away from the WZ-ZB phase transition region. In order to analyze the key electronic material properties (band gap, energetic position of the Fermi level, Fermi level pinning, etc.) in more detail, one needs to consider the tip-sample separation. In principle, the absence of tunneling current indicates the presence of a band gap. However, the width of the tunneling barrier increases with the tip-sample separation and thereby the transmission coefficient for electron tunneling decreases. As a result, the apparent band gap increases and the band edge positions cannot be determined due to insufficient sensitivity of the current measurement. In order to avoid this effect, we chose in the following a tip-sample separation that on the one hand is small enough for a sufficient sensitivity even within the band gap, but on the other hand is still well in the tunneling regime (no point contact).

Figure 2(d) shows such measured tunneling spectra. On the $\mathrm{ZB}$ segment, we observe the typical spectrum of 
GaAs(110): The valence band (VB) is seen at negative voltages and the conduction band $(\mathrm{CB})$ at positive voltages. ${ }^{29}$ Both bands are separated by an apparent band gap defined as the region where the tunneling current reaches the experimental noise level. Its width of about $1.5 \mathrm{eV}$ is consistent with the bulk band gap of GaAs. ${ }^{30}$ The Fermi level is found around mid-gap. In contrast to the $\mathrm{ZB}$ segment, the $\mathrm{WZ}$ segment shows a region with a tunneling current below the noise threshold or of low intensity that is wider $(1.9 \mathrm{eV})$. In addition, the Fermi level is close to the VB edge $\left(E_{\mathrm{V}}\right)$.

In order to understand the measured band gaps and positions of the Fermi level, we calculated the electronic structure of the ZB (110) and WZ (112̄0) GaAs surfaces. The density functional theory (DFT) calculations have been performed using the projector augmented wave approach as implemented in the VASP program $^{31}$ and the Heyd-Scuseria-Ernzerhof hybrid functional with a standard mixing parameter of 0.25 (HSE06). ${ }^{32}$ The calculated band gaps for zincblende and wurtzite GaAs are 1.33 and $1.30 \mathrm{eV}$, respectively. The equilibrium lattice parameters are found to be $a=5.69 \AA$ for the zincblende structure and $a=4.021 \AA, \quad c / a=1.6303$, and $\mathrm{u}=0.3753$ for the wurtzite crystal. The ZB (110) and WZ $(11 \overline{2} 0)$ surfaces have been modeled using slabs with 8 and 6 MLs thickness, respectively. The Ga and As atoms at the bottom side of the slab have been passivated with pseudohydrogens of 1.25 and 0.75 partial charges, respectively. The four topmost surface layers have been allowed to fully relax. The calculated surface band structures are shown in Fig. 3. We obtain bulk band gaps for $\mathrm{ZB} \mathrm{GaAs}$ rather close to the experimental values $[1.33 \mathrm{eV}$ vs. $1.42 \mathrm{eV}$ at room temperature $(1.51 \mathrm{eV}$ at $77 \mathrm{~K})]$. We note that the bulk band gap of the WZ phase $(1.30 \mathrm{eV})$ is found slightly smaller than that of the ZB phase $(1.33 \mathrm{eV})$. For both surfaces, the relaxation of the surface atoms pushes the surface states out of the fundamental bulk band gap, in agreement with the previous work. ${ }^{33}$ Hence the surface band gap is larger than the bulk band gap. Interestingly, the surface band gap of the WZ $a$-plane $(1.88 \mathrm{eV})$ is larger than the one of a ZB (110) plane $(1.5 \mathrm{eV})$. (a)

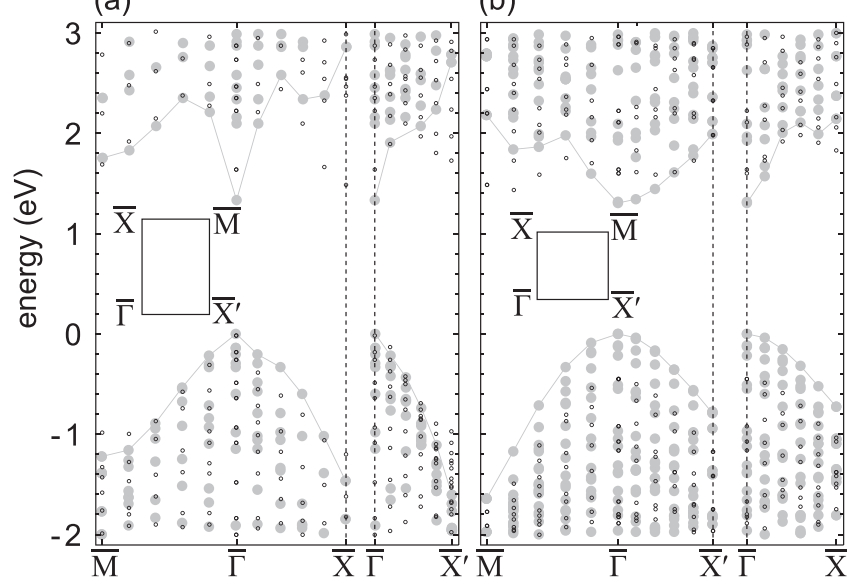

FIG. 3. Calculated surface band structures (open symbols) and projected bulk band structures (gray filled circles) of (a) ZB GaAs(110) and (b) WZ GaAs $(11 \overline{2} 0)$ surfaces. The gray lines indicate the projected bulk valence and conduction band edges. The energy scale is relative to the bulk valence band maximum. Insets: Schematic representation of the respective $2 \mathrm{D}$ Brillouin zones. The uppermost filled and lowermost empty surface states are both outside of the fundamental band gap.
Both surface band gaps are indirect. Note, the intrinsic surface states, being out of the fundamental band gap, do not induce any pinning of the Fermi level on both surfaces. As both the $\mathrm{WZ}$ and $\mathrm{ZB}$ segments are (equally) undoped, the question arises why different Fermi level positions are observed.

For undoped (or low-doped) GaAs with no intrinsic surface states in the band gap and no pinning of the Fermi level at the surface, tunneling spectra exhibit very large apparent band gaps (voltage region with no tunnel current), due to the large tip-induced band bending, which is not damped by screening carriers. ${ }^{34}$ As a result, for semiinsulating $\operatorname{GaAs}(110)$ with residual $n(p)$-type carriers, the branch at positive (negative) voltages in the tunneling spectra does not exist (below noise threshold) ${ }^{34}$ or is significantly shifted to much larger voltages. This effect is visibly not observed here. Instead we clearly observe both (VB and CB) branches of the tunneling current with normal intensities. This indicates that the Fermi level is pinned at both the WZ and the ZB surfaces.

This is further corroborated by the experimentally measured band gaps in the tunneling spectra, which agree very well with the surface band gaps. Since the surface states extend much farther into the vacuum, their contribution to the tunnel current is significantly larger than that of the bulk states. ${ }^{35}$ Thus the band edges observed in the tunneling spectra are determined primarily by the surface states. The observed band onsets and sizes of the gap also support the existence of a Fermi level pinning at both surfaces.

Upon closer examination, one can discern in the tunneling spectrum of the WZ structure GaAs, a weak current onset before the intensive conduction band onsets at about $+1.3 \mathrm{~V}$ (see gray arrow). In analogy, two weak onsets (almost in the noise level) occur at +0.6 and $-0.6 \mathrm{~V}$ for $\mathrm{ZB}$ GaAs (gray arrows). Such features were shown to arise from defect states at step edges ${ }^{29,36,37}$ suggesting a high step density.

At this stage, we address the origin of the Fermi level pinning. Since the intrinsic surface states of the ZB (110) and WZ $(11 \overline{2} 0)$ surfaces are outside of the band gap, the pinning of the Fermi level must arise from an extrinsic source, such as defects. We attribute the Fermi level pinning to the numerous step edges visible on the NW sidewalls. Steps on the GaAs(110) surface induce defect states in the band gap, which-when present at sufficiently large concentration-induce a pinning of the Fermi energy in midgap position. ${ }^{29,37}$ In our case, the steps have a concentration of $10^{5} \mathrm{~cm}^{-1}$ on the $\mathrm{ZB}$ segment. This corresponds to defect state concentration of $4 \times 10^{13} \mathrm{~cm}^{-2}$ assuming one defect state every lattice unit along the step edge. This high concentration is consistent with the observed defect current in the tunneling spectra and has been shown to induce a complete pinning of the Fermi level even in highly doped GaAs, and thus is a sufficient explanation for the undoped NWs studied here.

The step concentration on the WZ segment is in the same range. The steps are oriented preferentially along the [0001] direction on the WZ segment and have a large concentration of kinks. The defect states at step edges act as pinning centers. ${ }^{26}$ This indicates that on both polytype GaAs surfaces the defect states at the step edges are responsible for the Fermi level pinning. Based on the tunneling spectra and 
the theoretical calculations, we identify the ZB and WZ sidewall pinning levels to $E_{\mathrm{V}}+(0.7 \pm 0.1) \mathrm{eV}$ and $E_{\mathrm{V}} \pm 0.2 \mathrm{eV}$, respectively.

The presence of a Fermi level pinning at two different energies in the ZB and WZ segments has far reaching consequences for the NWs. The different pinning levels at the surface translate to extrinsic band offsets in the near surface region of the NW creating a $p-i$ junction. This extrinsic band offset will be screened toward the center of the NW by rearrangement of charges. For our undoped NW, the step induced pinning leads to an extrinsic band offset of about $0.7 \mathrm{eV}$ at the ZB-WZ interface in the near surface region. This offset is much larger than the intrinsic valence and conduction band offsets between $\mathrm{WZ}$ and $\mathrm{ZB}$ structure in bulk GaAs. ${ }^{18-20}$ Given this large difference and the high surface area to volume ratio of nanowires, the large sidewall pinning-induced extrinsic offsets will thus affect the carrier transport through the NW to a greater extent than the very small offsets between pure bulk GaAs WZ-ZB interfaces. Hence the interpretation of transport measurements requires a detailed assessment of the electronic properties and in particular of the Fermi pinning levels at the NW sidewalls.

The sidewall pinning induced offsets between WZ and ZB segments are not necessarily limited to our case of ultra clean NWs. The effect described here can be expected to be rather general, as the pinning levels of impurities and adsorbing atoms are likely different on WZ and ZB segments of the GaAs NWs or in general on the different polytype sidewall surfaces. Hence, extrinsic band offsets at ZB-WZ interfaces of NWs are likely to occur also under different environmental conditions (with varying energies, depending on the exact pinning levels). Thus, this effect needs to be taken into account for charge transport measurements and NW devices under different environmental conditions.

Note added in proof. During review of this paper, we became aware of a recent related study by M. Hjort et al., Ref. 38 .

This study was financially supported by the EQUIPEX program Excelsior, the European Community's Seventh Framework Program (Grant No. PITN-GA-2012316751, "Nanoembrace" Project) and the Impuls- und Vernetzungsfonds of the Helmholtz-Gemeinschaft Deutscher Forschungszentren under Grant No. HIRG-0014. T. Xu acknowledges the support from the National Natural Science Foundation of China (Grant No. 61204014).

${ }^{1}$ M. D. Pashley and K. W. Haberern, Phys. Rev. Lett. 67, 2697 (1991).

${ }^{2}$ E. F. Schubert, J. M. Kuo, R. F. Kopf, A. S. Jordan, H. S. Luftman, and L. C. Hopkins, Phys. Rev. B 42, 1364 (1990).

${ }^{3}$ P. M Fahey, P. B. Griffin, and J. D. Plummer, Rev. Mod. Phys. 61, 289 (1989).

${ }^{4}$ R. Ditchfield, D. Llera-Rodriguez, and E. G. Seebauer, Phys. Rev. B 61, 13710 (2000).

${ }^{5}$ J. Slotte, M. Gonzalez-Debs, T. F. Kuech, and J. C. Cederberg, J. Appl. Phys. 102, 023511 (2007).

${ }^{6} \mathrm{Ph}$. Ebert, S. Landrock, Y. P. Chiu, U. Breuer, and R. E. DuninBorkowski, Appl. Phys. Lett. 101, 192103 (2012).
${ }^{7}$ M. H. M van Weert, O. Wunnicke, A. L. Roest, T. J. Eijkemans, A. Yu Silov, J. E. M. Havekort, G. W. 't Hooft, and E. P. A. M. Bakkers, Appl. Phys. Lett. 88, 043109 (2006).

${ }^{8}$ D. Wang, Y.-L. Chang, Q. Wang, J. Cao, D. B. Farmer, R. G. Gordon, and H. Dai, J. Am. Chem. Soc. 126, 11602 (2004).

${ }^{9}$ S. Zhang, E. R. Hemesath, D. E. Perea, E. Wijaya, J. L. Lensch-Falk, and L. J. Lauhon, Nano Lett. 9, 3268 (2009).

${ }^{10}$ R. E. Algra, M. A. Verheijen, M. T. Borgström, L.-F. Feiner, G. Immink, W. J. P. van Enckevort, E. Vlieg, and E. P. A. M. Bakkers, Nature 456, 369 (2008).

${ }^{11}$ P. Caroff, K. A. Dick, J. Johansson, M. E. Messing, K. Deppert, and L. Samuelson, Nat. Nanotechnol. 4, 50 (2009).

${ }^{12}$ K. A. Dick, C. Thelander, L. Samuelson, and P. Caroff, Nano Lett. 10, 3494 (2010).

${ }^{13}$ S. Assali, I. Zardo, S. Plissard, D. Kriegner, M. A. Verheijen, G. Bauer, A. Meijerink, A. Belabbes, F. Bechstedt, J. E. M. Haverkort, and E. P. A. M. Bakkers, Nano Lett. 13, 1559 (2013).

${ }^{14}$ S. A. Dayeh, D. Susac, K. L. Kavanagh, E. T. Yu, and D. Wang, Adv. Funct. Mater. 19, 2102 (2009).

${ }^{15}$ N. Akopian, G. Patriarche, L. Liu, J.-C. Harmand, and V. Zwiller, Nano Lett. 10, 1198 (2010).

${ }^{16}$ C. Thelander, P. Caroff, S. Plissard, A. W. Dey, and K. A. Dick, Nano Lett. 11, 2424 (2011).

${ }^{17}$ A. De and C. E. Pryor, Phys. Rev. B 81, 155210 (2010).

${ }^{18}$ M. Heiss, S. Conesa-Boj, J. Ren, H.-H. Tseng, A. Gali, A. Rudolph, E. Uccelli, F. Peiró, J. R. Morante, D. Schuh, E. Reiger, E. Kaxiras, J. Arbiol, and A. Fontcuberta i Morralet, Phys. Rev. B 83, 045303 (2011).

${ }^{19}$ P. Kusch, S. Breuer, M. Ramsteiner, L. Geelhaar, H. Riechert, and S. Reich, Phys. Rev. B 86, 075317 (2012).

${ }^{20}$ R. Gurwitz, A. Tavor, L. Karpeles, I. Shalish, W. Yi, G. Seryogin, and V. Narayanamurti, Appl. Phys. Lett. 100, 191602 (2012).

${ }^{21}$ S. Plissard, K. A. Dick, X. Wallart, and P. Caroff, Appl. Phys. Lett. 96, 121901 (2010).

${ }^{22}$ A. M. Munshi, D. L. Dheeraj, J. Todorovic, A. T. J. van Helvoort, H. Weman, and B.-O. Fimland, J. Cryst. Growth 372, 163 (2013).

${ }^{23}$ T. Xu, K. A. Dick, S. Plissard, T. H. Nguyen, Y. Makoudi, M. Berthe, J.-P. Nys, X. Wallart, B. Grandidier, and P. Caroff, Nanotechnology 23, 095702 (2012).

${ }^{24}$ T. Xu, J.-P. Nys, A. Addad, O. I. Lebedev, A. Urbieta, B. Salhi, M. Berthe, B. Grandidier, and D. Stiévenard, Phys. Rev. B 81, 115403 (2010).

${ }^{25}$ S. Plissard, K. A. Dick, G. Larrieu, S. Godey, A. Addad, X. Wallart, and P. Caroff, Nanotechnology 21, 385602 (2010).

${ }^{26}$ B. Siemens, C. Domke, Ph. Ebert, and K. Urban, Phys. Rev. B 59, 3000 (1999).

${ }^{27}$ E. Hilner, U. Håkanson, L. E. Fröberg, M. Karlsson, P. Kratzer, E. Lundgren, L. Samuelson, and A. Mikkelsen, Nano Lett. 8, 3978 (2008).

${ }^{28} \mathrm{H}$. Eisele and Ph. Ebert, Phys. Status Solidi (RRL) 6, 359 (2012).

${ }^{29}$ S. Gaan, R. M. Feenstra, Ph. Ebert, R. E. Dunin-Borkowski, J. Walker, and E. Towe, Surf. Sci. 606, 28 (2012).

${ }^{30}$ G. Brammertz, Y. Mols, S. Degroote, V. Motsnyi, M. Leys, G. Borghs, and M. Caymax, J. Appl. Phys. 99, 093514 (2006).

${ }^{31}$ G. Kresse and J. Hafner, Phys. Rev. B 47, 558 (1993); G. Kresse and J. Furthmüller, ibid. 54, 11169 (1996).

${ }^{32}$ J. Heyd, G. E. Scuseria, and M. Ernzerhof, J. Chem. Phys. 118, 8207 (2003); J. Heyd, G. E. Scuseria, and M. Ernzerhof, ibid. 124, 219906 (2006).

${ }^{33}$ B. Engels, P. Richard, K. Schroeder, S. Blügel, Ph. Ebert, and K. Urban, Phys. Rev. B 58, 7799 (1998).

${ }^{34}$ N. D. Jäger, Ph. Ebert, K. Urban, R. Krause-Rehberg, and E. R. Weber, Phys. Rev. B 65, 195318 (2002).

${ }^{35}$ Ph. Ebert, S. Landrock, Y. Jiang, K. H. Wu, E. G. Wang, and R. E. DuninBorkowski, Nano Lett. 12, 5845 (2012).

${ }^{36}$ L. Ivanova, S. Borisova, H. Eisele, M. Dähne, A. Laubsch, and Ph. Ebert, Appl. Phys. Lett. 93, 192110 (2008); Ph. Ebert, S. Schaafhausen, A. Lenz, A. Sabitova, L. Ivanova, M. Dähne, Y.-L. Hong, S. Gwo, and H. Eisele, ibid. 98, 062103 (2011).

${ }^{37}$ A. Sabitova, Ph. Ebert, A. Lenz, S. Schaafhausen, L. Ivanova, M. Dähne, A. Hoffmann, R. E. Dunin-Borkowski, A. Förster, B. Grandidier, and H. Eisele, Appl. Phys. Lett. 102, 021608 (2013).

${ }^{38}$ M. Hjort, S. Lehmann, J. Knutsson, R. Timm, D. Jacobsson, E. Lundgren, K. A. Dick, and A. Mikkelsen, Nano Lett. 13, 4492 (2013). 